\title{
Von einer Poetik der Drastik zum Entwurf eines „Realismus der Globalisierung" am Beispiel der Romane von Terézia Mora
}

\author{
From a poetics of the drastic to a sketch of a realism of globalization illustrated by Terézia \\ Mora's novels
}

\begin{abstract}
In light of this (not so) new century, there is good reason to consider the rise of a »realism of globalization «. As the former leading agent of modelling alternative worlds, the tradition of the realistic novel has long lost ground against the dominance of new, »visual novels « (i.e. The Wire) with their own approach towards realistic aesthetics. What is the role that literature can play in this transformative, mediatic field? Which originality can it claim?

The objective of this (two part) study is to respond to these concerns by example. The case is made for two novels, Alle Tage (2004) and Der einzige Mann auf dem Kontinent (2009), by the GermanHungarian writer Terézia Mora, which might function as a showcase for the ways in which contemporary fiction responds to and contributes in shaping the discourse of globalization. The first part of this study is presented hereby.
\end{abstract}

Key words: globalization; migration; realism; poetics; Terézia Mora

Zusammenfassung: Wir können unter den Vorzeichen des nicht mehr ganz so jungen aktuellen Jahrhunderts von einem »Realismus der Globalisierung « sprechen. Doch scheint der Erzähltext, im speziellen der Roman als Leitmedium von alternativen Weltentwürfen, gegen die Dominanz der visuellen Narrative (z.B. TV-Serien wie The Wire) im Sinne einer zeitgenössischen Ästhetik des Realen eine seiner letzten Domänen zu verlieren. Welche Rolle kann die Literatur angesichts dieser weit fortgeschrittenen Verschiebung des medialen Feldes noch spielen? Welche Eigenständigkeit kann sie darin behaupten?

Das Ziel einer in zwei Teilen konzipierten Untersuchung soll es sein, diese beiden Fragen am Beispiel der Romane Alle Tage (2004) und Der einzige Mann auf dem Kontinent (2009) von Terézia Mora exemplarisch und - mit Blick auf die Hauptthese auch experimentell für die Literatur - zu beantworten. Der erste Teil dieser Untersuchung, in welcher der Roman Alle Tage im Zentrum steht, liegt hiermit in einer ersten Fassung vor.

Stichwörter: Globalisierung; Migration; Realismus; Poetik; Terézia Mora

*M.A., Universität Potsdam, Institut für Romanistik. Email: kraft@uni-potsdam.de 


\section{Kraft, T. - Poetik der Drastik}

\section{Einleitung}

War der literarische Realismus des 19. Jahrhunderts ein »bürgerlicher« und der des 20. ein »sozialistischer«, so können wir unter den Vorzeichen des nicht mehr ganz so jungen aktuellen Jahrhunderts von einem »Realismus der Globalisierung « sprechen ${ }^{1}$.

Das Leitmedium dieses Realismus des 21. Jahrhunderts ist nicht mehr der »klassische « Roman, die Malerei oder die Bildhauerei, sondern der Film, und in besonderem Maße die avantgardistischen Serienformate des Fernsehens. Der Rückbezug zur generischen Form des Romans bleibt bei diesen »visual novels« jedoch stets evident, wie hochwertige, vor allem von den US-amerikanischen networks verantwortete Produktionen wie The Wire (2002-2008) oder Breaking Bad (2008-2012) seit Jahren sehr erfolgreich unter Beweis stellen. Der Erzähltext als Medium einer zeitgenössischen Ästhetik des Realen scheint gegen diese Dominanz der visuellen Narrative eine seiner letzten Domänen zu verlieren. Welche Rolle kann die Literatur angesichts dieser weit fortgeschrittenen Verschiebung des medialen Feldes noch spielen? Welche Eigenständigkeit kann sie darin behaupten?

Das Ziel dieser in zwei Teilen konzipierten Untersuchung soll es sein, diese beiden Fragen am Beispiel der Romane Alle Tage (2004) und Der einzige Mann auf dem Kontinent (2009) von Terézia Mora exemplarisch nachzugehen. Der erste Teil dieser Untersuchung, in welcher der Roman Alle Tage im Zentrum steht, liegt hiermit in einer ersten Fassung vor.

\section{Merkmale einer beschleunigten Globalisierung}

Wollen wir unsere Jetztzeit als die vorerst jüngste von bisher vier Phasen beschleunigter Globalisierung (ETTE 2012: 8-26, 37) beschreiben, so können mindestens vier Großkomplexe als zentrale Aspekte dieser Phase angenommen werden: 1) Migration und Transkulturationsprozesse im weltweiten Maßstab, 2) die globalen Finanz- und

\footnotetext{
1 Zur literaturhistorisch wie literarästhetisch und -theoretisch viel diskutierten Konzeption des oder eines »Realismus« sei hier nur bereits soviel gesagt: Realismus wird im Folgenden verstanden als (und damit: reduziert auf einen) Epochen- und Stilbegriff. Vgl. für einen kompakten (und auf die Germanistik fokussierten) Forschungsüberblick zum Realismus als Epochenbegriff anhand möglicher Darstellungskriterien AUST 2000: 17. Beim Epochenbegriff Realismus gehe ich von der Annahme aus, dass in diesem (zweifellos unscharfen) Terminus in seiner spezifisch historischen Konstellation des 19. Jahrhunderts die Möglichkeit eines epistemischen Transfers in unsere Jetztzeit angelegt ist. Als Folge dieser Übertragung soll es im Folgenden0 daher um einen literarischen Stil gehen, den wir im Sinne Dietmar Daths als eine Poetik der „Drastik“ (DATH 2005) begreifen können.
} 


$$
\text { Kraft, T. - Poetik der Drastik }
$$

Wirtschaftskrisen als spätkapitalistische Vorzeichen eines ökonomischen und ideologischen Systemwandels, 3) das Weltklima als ökologische und energiepolitische Kernfrage globaler Verteilungskämpfe um Lebensraum und natürliche Ressourcen, sowie 4) der endgültige Eintritt der Menschheit ins Digitale Zeitalter.

Ähnlich fassen es die Antragssteller des an der LMU München angesiedelten und im April 2012 angelaufenen DFG-Graduiertenkollegs »Funktionen des Literarischen in Prozessen der Globalisierung«, wenn sie den Problemhorizont des Begriffes Globalisierung im Kontext seiner möglichen fiktionalen Ausgestaltungen in folgende Teilbereiche gliedern: a) das weltweit agierende Finanzkapital, seine Protagonisten und Mechanismen, b) die globalisierungskritischen Bewegungen, c) die Omnipräsenz von global vermarkteten Waren, d) die Reorganisation und diskursive Verhandlung kultureller Grenzen sowie e) die aus der Tradition des 17. und 18. Jahrhunderts aktualisierte Erprobung des kosmopolitischen Blicks (vgl. STOCKHAMMER 2011: 11).

Moras Romane lassen sich in zweierlei Weise einem hier postulierten »Realismus der Globalisierung« zuordnen. Zum einen durch die ganz in die gegenwärtigen Gesellschaftskonfigurationen des Globalen verankerten Romanthemen: der Migration als Zustand fortgesetzter Ortlosigkeiten (Alle Tage) sowie der internet-basierten Arbeitsökonomie (Der Mann...). Zum anderen durch die literarische Darstellung der von diesen Konstellationen dynamisierten und im Modus der Literatur experimentell erprobten Weltwahrnehmungen als Krise von Sprache und Identifikation sowie als Verlust bindender Referentialitäten. So unterschiedlich beide Romane in ihrer thematischen Ausrichtung auf den ersten Blick auch sein mögen, so deutlich fügen sie sich ein in einen gemeinsamen, den Herausforderungen globalisierter Lebensumstände verarbeitenden Rahmen. Die Spezifik einer „Literatur des Globalen“ (REICHARDT 2010), wie Mora sie hier entwickelt, ist im Wesentlichen eine Leistung ihrer Erzähltechnik und Gattungskolportage. Darin verbinden und überlagern sich Erzähl- und Personenstimmen in komplexer Polyphonie und erzeugen Hochgeschwindigkeitsnetze variabler Perspektiven-, Zeit- und Distanzverhältnisse zwischen Erzähler- und Figurendiegese, welche die Differenz zwischen Erlebtem und Erzähltem, zwischen Erfahrung und Vermittlung in zuweilen berauschender Prosa nachvollziehen. Diese Differenz jedoch ist Fundamentalerfahrung der Protagonisten migratorischer wie postmigratorischer Gesellschaftskontexte, sie ist zweite Natur eines Berufsalltags im Digitalen Zeitalter. Darin leisten Moras Texte eine spezifisch literarische Reflexionsarbeit als Produktionsorte von „narratives of globalization“ (GUPTA 2009: 63ff.), literarische Beiträge 


$$
\text { Kraft, T. - Poetik der Drastik }
$$

$\mathrm{zu}$ einer diskursiven Arbeit am Begriff und der Vorstellung von »Globalisierung «. Denn jenseits der Tatsache, dass beide Romane ihre Handlung und Figuren vor dem thematischen Hintergrund der genannten Globalisierungsprozesse entfalten, entwickeln sie eine literarische Kritik geläufiger Vorstellungen von Globalisierung, die es im weiteren noch zu entwickeln gilt.

\section{Terézia Mora}

Terézia Mora ist trotz ihres nicht sehr umfangreichen Euvres - bisher liegen von ihr neben Hörspielen und Theaterstücken ein Erzählband sowie zwei Romane vor - sicherlich eine der profiliertesten Autorinnen ihrer Generation. Folgen wir Ilija Trojanow, dann ist sie eines der prominentesten Beispiele nicht für eine Migranten- oder »Chamisso-Literatur«, wie es im germanistischen Umfeld der Robert-Bosch-Stiftung häufig heißt, sondern für „das Hineinwachsen der deutsch-sprachigen Literatur ins Weltliterarische mit Hilfe der Agenten der Weltläufigkeit und Mehrsprachigkeit“" (TROJANOW in HÜBNER 2010: 18). Eine solche Agentin ist die deutsch-ungarische Autorin Terézia Mora.

Ich bin eine Osteuropäerin, eine Frau, ich komme aus archaischen Verhältnissen, Erzkatholizismus und realer Kommunismus waren das K.u.K. meiner Kindheit - wo, was wäre ich heute, wenn ich zimperlich wäre: nirgends, nichts.

In Wahrheit ist - natürlich - das Gegenteil der Fall. Alles greift mich an. Ich bin nur insofern nicht zimperlich, dass ich darüber nicht traurig, sondern wütend werde. Ich bin empört. Dann schreibe ich. 'Kunst hat kein Ziel, sondern einen Grund', behauptete Lajos Kassák. Das ist meiner. (MORA 2006: 68-69)

Gegenüber ihrem ersten Verlag Rowohlt, der Mora nach Gewinn des Open-MikeLiteraturwettbewerbs 1997 einen Vertrag anbietet, stellt sie sich zuerst mit ihrem Ehe-Namen vor,

einem kerndeutschen Namen, [...] aber ich wollte nicht unter dem Namen meiner Schwiegermutter veröffentlichen. [...] Mora ist übrigens auch nicht mein richtiger Name. Ich wollte einen Namen wählen, dem man nicht sofort anhört, wo er herkommt. Ich wollte mich maskieren. Ich bin zu hochnäsig, um einen Trend auszunutzen. Ich wollte die Wahrheit erfahren: Man sollte nicht wissen, wer diesen Text geschrieben hat, aber diesen Text dennoch als gültig erkennen. (MORA in AYATA u. a. 2005: 30) 


$$
\text { Kraft, T. - Poetik der Drastik }
$$

In einer von ihr verfassten Europa-Kolumne zum EU-Beitritt mitteleuropäischer Länder verlegt sie ihre Herkunft soweit in die (historische) Vergangenheit, dass sich eine Frage nach der originären Eingrenzung ihrer Identität erübrigt.

Nachdem mein erstes Buch mit Erzählungen aus der österreichisch-ungarischen Grenzregion erschienen war, sagte ich, um der Festlegung zu entgehen, ob ich nun eine ungarische oder eine deutsche Schriftstellerin sei, dass ich mich als Pannonierin verstünde. Das war eine gute Antwort, darauf konnte jeder nur nicken. (MORA in KRAFT 2007: 25)

Das „Außenseiterleben” (WEIDERMANN 2004: 27) ihrer Kindheit ist der Erfahrungsschatz, von dem sie in den dunklen, von den Entsagungen einer (deutsch-)feindlichen und archaischen Dorf-Welt erzählenden Geschichten ihrer ersten Publikation Seltsame Materie (1999) ein beklemmendes Zeugnis abgibt. Hier, im Grenzgebiet zwischen drei Staaten, zwischen Ost- und Westeuropa in der Endphase des Kalten Krieges, paart sich die deprimierende Erfahrung einer unentrinnbaren Dörflichkeit aller Lebensabläufe mit der stetigen Präsenz des Unbekannten durch die politisch-territoriale Grenze. Sie ist allgegenwärtiger Fluch und Projektionsfläche innerster Sehnsüchte, die in Gewalt oder Alkohol ertränkt werden: „alles ist hier Grenze“ (MORA 2005: 58). Diese Grenzfiktionen erzählen von einer Heimat, auf der ein Tabu liegt. Ihr prekäres Wesen als Ort der Unruhe und des Untergangs wird verstärkt durch das mütterliche Verbot: „Erzähl ja niemandem, wie es passiert ist. Und erzähl auch sonst nichts von hier“ (MORA 2005: 9). Das Erzählbare wird im desaströsen hier der Heimat zu einer eigenen Grenze zwischen Sag- und Unsagbarem.

Der mütterliche Befehl an die Protagonistin, nur ja nichts von hier zu erzählen, wird durch die Erzählung selbst missachtet (denn es wird ja erzählt) und lässt sich auch als knapper Kommentar der Autorin zu den Debatten der Literaturkritik und -wissenschaft in den neunziger Jahren lesen, in denen kontrovers die Qualität zeitgenössischer deutschsprachiger Literatur vor dem Hintergrund einer Renaissance des Erzählens diskutiert wurde (vgl. hierzu STOPKA 2001: 147-151 und allgemeiner HARDER 2001). In Alle Tage greift Mora diese reichlich überladene Diskussion in einer kurzen aber deftigen Parodie wieder auf: „Blablablabla, sagt der Linke. Ein wahrhaft tragisches Schicksal! Und so realistisch beschrieben! Sterzergreifend! Ich kann’s nicht mehr hören, scheiß neue Lust am Erzählen!“ (MORA 2004: 388). 
Kraft, T. - Poetik der Drastik

\section{"Drastik": zu Moras literarischer Programmatik}

Wer seine Romanfiguren so dezidiert auf die konventionellen Urteile literaturwissenschaftlicher wie feuilletonistischer Publizistik schimpfen lässt, der hat mit realistischem Erzählen im Sinne einer naiven Abbildungsästhetik oder nüchternen und alltagsgesättigten Großstadtprosa wenig zu schaffen. Was kann es dann heißen, von Terézia Mora im Zusammenhang von Überlegungen zum literarischen Realismus im 21. Jahrhundert zu sprechen?

Zur Beantwortung dieser Frage benötigt es eines kleinen Umweges, der zu einem Interview mit dem FAZ-Journalisten und Schriftsteller Dietmar Dath in der Hildesheimer Literaturzeitschrift bella triste führt. Hier konkretisiert Dath den von ihm an anderer Stelle geäußerten Anspruch, mit Literatur nicht das Wirkliche, sondern , das Wahre' fokussieren zu wollen:

Mit der 'Wahrheit' [...] ist dasjenige vom Denkbaren gemeint, was an Emanzipation vom Naturzusammenhang und nur Vorgefundenen [...] dem gegenwärtigen, wirklichen Zustand bereits implizit ist [...]. [D]iese Dinge durchzusetzen, kann Kunst (also auch Literatur) helfen, indem sie entwickelt, wie es wäre, wenn sie durchgesetzt wären, oder wie es kommen muß, wenn sie nicht durchgesetzt werden [...] (DATH in KAROW, WINKLER 2006: 64-65)

Diese „spekulative Phantastik“, wie Dath schreibt, steht in einer ästhetischen Tradition, die man »Ermöglichungsrealismus « nennen könnte. Der hier aufscheinende Wahrheitstopos ${ }^{2}$ lässt sich mühelos in die Tradition des französischen, empiristischen Realismus des 19. Jahrhunderts stellen und findet sich mit Blick auf die Rolle, die der Literatur als soziale Veränderung herbeiführende make-believe-Maschine zugesprochen wird, ebenso in der sozialemanzipatorischen Realismusagenda der russischen revolutionären Demokraten der 1920er Jahre wieder (vgl. KLEIN 2010: 165-167; 174-177). Zugleich sieht Dath eine Parallele zwischen einer Renaissance realistischer Ästhetik (z.B. im Sinne einer Verbindung von Wissenschaft, Lebenswelt und Kunst) und einer Wiederkehr sozialhistorischer Probleme in einer Form, wie sie schon das 19. Jahrhundert kannte:

[W]enn man - so wie ich - glaubt, daß wir mit den Problemen jenes Jahrhunderts noch nicht fertig sind, sondern diese vielmehr jetzt, nach einer Art Auszeit im zwanzigsten,

\footnotetext{
${ }^{2}$ Paradigmatisch steht hierfür Stendhals Motto „La verité, la verité“, mit dem Le Rouge et le Noir beginnt. Vgl. hierzu sowie zum Wahrheitstopos bei Stendhal DETHLOFF 1997: 80ff..
} 


$$
\text { Kraft, T. - Poetik der Drastik }
$$

als globale kapitalistische Zustände (so rein damals nur in England, jetzt fast überall) gerade in full force zurückkehren, dann muß man entsprechend vorgehen. (DATH in KAROW, WINKLER 2006: 64-65)

Damit spricht Dath vom Realismus ohne ihn so zu nennen, verstand sich dieser doch als ästhetische Antwort auf die industriellen, politischen und sozialen Revolutionen des 19. Jahrhunderts. Folgt man Peter Brooks, dann war jenes Jahrhundert

an age where history takes on new importance, and learns to be more scientific, and where theories of history come to explain how we got to be how we are, and in particular how we evolved from earlier forms to the present. It is the time of industrial, social, and political revolution, and one of the defining characteristics of any realist writing is I think a willingness to confront these issues. (BROOKS 2005: 13)

Dietmar Dath nennt das „Drastik“ (Vgl. auch DATH 2005). Drastik ist eine ästhetische Antwort auf diese Zustände unserer Zeit. Drastik ist die literarische - und mithin realistisch zu nennende - „willingness to confront“. Terézia Mora ist dieser unmittelbar politische Impetus in der Herausbildung einer eigenen literarischen Ästhetik nicht fremd. Der poetologische Dialog zwischen Dath und Mora findet aber nicht an sondern vielmehr neben dieser politischen Frage statt. Bei Terézia Mora hat Drastik einen noch deutlicher als bei Dath akzentuierten wirkungsästhetischen Anspruch: „Kunst muss, nach Moras Definition, weit genug gehen. [...] Weit genug zu gehen heißt, jemanden zu berühren.“ (MORA 2006: 69) Diese Wirkung schließlich stellt die außerliterarische Referenz her und funktioniert im Sinne von Lotmans Kunstbegriff als „,sekundäres modellbildendes System“ (LOTMANN 1972: 22ff.) im Modus einer rezeptionsästhetischen »Möglichkeitsmaschine«:

Wenn wir von Drastik in der Literatur sprechen, dann meinen wir jenen Versuch, etwas zu generieren, das eine ähnliche Wirkung entfaltet, wie die Wirklichkeit (bisher: das Leben). [...] Zur Hilfe kommt uns dabei, dass ein 'es-selbst-erlebt-Haben' nicht notwendig ist, um zu verstehen, was einem da erzählt wird. [...] Zudem neigen wir dazu, uns von Ereignissen persönlich betroffen zu fühlen, obwohl wir gar nicht persönlich gemeint sind. Dieses Selbstbezogensein des Menschen ist etwas, das sich Literatur, die auf das Engagement des Lesers angewiesen ist, zu Nutze macht. [...] Mit Drastik versuchen wir den Punkt zu treffen, an dem die wie auch immer praktizierte Indifferenz nicht mehr aufrecht zu erhalten ist [...]: mit Drastik drücken wir unser Unbehagen aus, Unbehagen ist ein zentraler Grund für und eine Funktion von Kunst. (MORA 2006: 70-71)

Ein drastischer Satz ist „nicht der [...], in dem Blut und Eiter spritzen oder einer, in dem es von Scheiße und Fotze wimmelt. [...] Ein wahrhaft drastischer Satz ist einer, der mir keine Chance lässt, die in ihm enthaltene Wahrheit zu leugnen“ (MORA 2006: 74). Auch hier lässt 


\section{Kraft, T. - Poetik der Drastik}

sich der Verweis auf eine Tradition gesellschaftskritisch engagierter ${ }^{3}$, bzw. sozialrevolutionärer realistischer Literatur kaum leugnen, die sich - nicht ohne Emphase auflehnt gegen eine als empörend empfundene soziale Wirklichkeit. Natürlich heißt das heute nicht mehr Literatur an die Unterprivilegierten, gegen die Sozialnormen eines herrschenden Gesellschaftssystems oder für eine (kultur-)politische Ideologie zu schreiben. Und dennoch scheinen wir bei Mora (wie bei Dath) Beispiele für eine Autorschaft des 21. Jahrhundert zu erkennen, die sich nicht der naiven Vorstellung postideologischer Zeiten hingibt, sondern sich unter den Vorzeichen eines neu zu artikulierenden Realismusbegriffs durchaus als politisch engagiert begreift. Das würde die Einschätzung Wolfgang Kleins aufgreifen, der als Antwort auf die postmoderne Kritik am Wirklichkeits- und Repräsentationsbegriff konstatiert:

Der analytische Gehalt der heutigen Argumentationen scheint in früheren Realismusbegriffen des öfteren bereits positionsbildend auf. Das läßt vermuten: Auch durch die heutigen Bedenken ist 'Realismus' nicht erledigt. (KLEIN 2010: 153)

Bei Mora ist diese Konturierung eines neuen Realismus-Begriffs ein Ergebnis der Sprache. Das ist zunächst keine Überraschung, sondern vielmehr einzig verbliebene ästhetische Option in der Auseinandersetzung mit einer außerliterarischen Wirklichkeit und angesichts eines durch den linguistic turn propellierten Erkenntnisstandes, der „mit der Konzeption von der Vorgängigkeit einer Welt auf[räumt], die vom Kunstwerk mimetisch gespiegelt wird“ (RECKWITZ 2008: 610). Gerade Alle Tage widersetzt sich zudem jeder naiven Lesart, die den Roman nach eindeutiger Referentialität absucht um dann in einem zweiten Schritt daran seine Plausibilität als quasi-historischen Roman eines postsowjetischen Bürgerkriegsflüchtlings, der aus Jugoslawien nach Berlin kommt, ablesen zu können.

\section{Synopse zu Alle Tage}

Terézia Moras Debütroman Alle Tage, 2004 im Luchterhand Literaturverlag erschienen, ist nach dem Erzählband Seltsame Materie ihre zweite von mittlerweile drei literarischen Monographien und wurde begeistert von der deutschsprachigen Literaturkritik aufgenommen:

\footnotetext{
${ }^{3}$ In der germanistischen Diskussion um den literturhistorischen Realismusbegriff gilt die Herausbildung einer kritischen Literaturfunktion in der Vormärzzeit als wichtige Voraussetzung für den »eigentlichen « Realismus der zweiten Hälfte des 19. Jahrhunderts, auch wenn dieses Urteil z.B. von Friedrich Sengle angezweifelt wird (Aust 2000: 56ff.).
} 


\section{Kraft, T. - Poetik der Drastik}

„Man liest und ist berauscht. Avantgarde und Genuss passen ausnahmsweise einmal zusammen”, schreibt z.B. Jörg MAGENAU in der taz (2004: 9). Von ihrem Erfolg sprechen auch die zahlreichen Auszeichnungen und Förderpreise: Allein für Alle Tage hat Terézia Mora bisher fünf zum Teil hochdotierte Literaturpreise verliehen bekommen ${ }^{4}$.

»Alle Tage«, so heißt auch ein Gedicht aus Die gestundete Zeit, dem Lyrikband, mit dem Ingeborg Bachmann 1953 schlagartig berühmt wurde. Steht Bachmanns Text noch ganz unter dem Eindruck einer beginnenden atomaren Aufrüstung im Zeichen des Kalten Krieges, setzt Moras Roman in den frühen postsozialistischen Jahren an und spricht von einem Held, der, wie bei Bachmann, „den Kämpfen fern [bleibt]” (BACHMANN 1985: 28).

Der Roman erzählt die Geschichte von Abel Nema, der nach der Abiturfeier aus seiner Heimat, in der gerade ein Bürgerkrieg ausbricht, flüchtet und von Ungarn aus über einen Hinweis seiner Mutter in eine deutsche Stadt gelangt, die im Roman nur B. heißt. Nicht in Berlin, sondern „in B. Er solle es in B. versuchen” (MORA 2004: 74), heißt es im Roman. Gebeutelt von einem schweren Gasunfall, den er nur knapp überlebt, ist dem bis dahin mehr oder weniger dahin lebenden Abel mit einem Mal ein besonderes Sprachtalent gegeben, das Abels zukünftigen Weg ebenso prägen wird wie eine vormals unbekannte Orientierungsschwäche.

[A]ls er dann endlich wieder zu sich gekommen war, gab es allerlei Symptome. Es fing damit an, dass er sich auf dem Weg von der Toilette zurück in das Krankenzimmer verlief und - wie lange? - in den Krankenhausfluren herumirrte, bis Bora ihn fand. [...] Ein weiteres Symptom war, ist, dass er den Zettel ruhig hätte wegwerfen können oder sich erst gar nichts aufschreiben brauchen, er hatte sich den Namen sofort gemerkt und wusste auch, dass er ihn nicht mehr vergessen würde, weil er ab jetzt nichts, was Sprache und memorisierbar ist, jemals wieder vergessen würde. (MORA 2004: 85)

Was Abel so am Beginn seiner Flucht- und Migrationsgeschichte an Orts- und Orientierungswissen einbüßt, fällt ihm an Sprachfähigkeit und Gedächtnisleistung zu. Anstatt Orte zu erinnern, memorisiert er Wörter. Das Fluidum der Sprache(n) tritt an die Stelle der Verlässlichkeit und Stabilität des Räumlichen.

\footnotetext{
${ }^{4}$ Förderpreis für Literatur der Akademie der Künste Berlin 2004, Preis der LiteratourNord 2004, Mara-CassensPreis 2004, den Preis der Leipziger Buchmesse 2005, sowie den Literaturpreis der Stadt Graz 2007, darüber hinaus 2010 für ihr bisheriges Werk den Adelbert-von-Chamisso-Preis und den Erich Fried Preis (vgl. die Autorenseite ihres Verlages unter http://www.randomhouse.de/author/author.jsp?per=77303 (01/08/2012)). 2011 erhielt sie den Übersetzerpreis der Kulturstiftung NRW für die Übersetzung von Esterházys Ein Produktionsroman sowie für ihr bisheriges übersetzerisches Lebenswerk (!) (vgl. http://www.boersenblatt.net/431591/ (01/08/2012)).
} 


$$
\text { Kraft, T. - Poetik der Drastik }
$$

In B. angekommen, organisiert ihm Professor Tibor, sein zukünftiger Mentor, ein Stipendium, mit dem er in den kommenden Jahren einsam im Sprachlabor der Universität zehn verschiedene Sprachen bis zur absoluten Perfektion lernt. Über Tibor macht Abel Bekanntschaft mit dessen Assistentin Mercedes und ihrem Sohn Omar. Nach vier Jahren in einer verdreckten Studenten-WG mit dem theatralischen und hyperaktiven Konstantin Tóti zieht er nach „Anarchia Kingania“, ein herunter gekommenes Dachgeschoss ohne Heizung, in dem die exzentrische und laute Kinga lebt, die Abel zusammen mit ihrer Band bereits auf seiner Zugfahrt nach B. kennen gelernt hatte:

Sie lachte. Keiner ihrer Zähne berührte seinen Nachbarn. Brombeerfarbene Lippen unter großen, behaarten Nasenlöchern, dafür großartige Wangenknochen, Augen, Stirn, darüber ein Durcheinander nie gekämmter dunkler Locken. Hörte nicht auf zu lachen. (MORA 2004: 135)

Auf dem Rückweg einer Reise mit Kingas Band trifft er zufällig erneut auf Mercedes. Sie wird Abels Leben, zu jenem Zeitpunkt ein richtungsloses Chaos zwischen Eskapismus und Apathie, wieder Ordnung geben, zumindest ordentliche Papiere. Mercedes, „ihr Name ist Gnade“ (MORA 2004: 407), wird Abel, den fahnenflüchtigen Migranten mit prekären Wohnverhältnissen, die Möglichkeit geben zu heiraten und in Scheinehe zu leben. Das Zweckpaar heiratet, doch für Mercedes, die sich erst spät eingestehen wird, jenen unerklärlichen Mann zu lieben, interessiert sich Abel kaum. Nur Omar, Mercedes' Sohn, scheint diesem Fremden ohne festen Wohnsitz etwas zu bedeuten. Doch auch der legalisierte Aufenthalt kann Abels Leben nicht beruhigen, im Gegenteil: Seine schäbige Wohnung unterm Dach verwahrlost zunehmend, seine letzten Beziehungen zu anderen Menschen brechen ab, und nachdem Mercedes Abel in flagranti mit einem Lustknaben erwischt, ist ihm auch der Kontakt zu Omar untersagt. Schließlich kommt es, nach einer Fußverletzung, einem mehrtätigen Drogenrausch, dem Verlust seiner Papiere und einer unheilvollen Begegnung mit jugendlichen Schlägern zur Katastrophe, die Abel nur knapp überlebt. Als Folge der Drogenexzesse sowie seiner schweren Verletzungen wird sein Sprachgedächtnis fast gänzlich ausgelöscht. Einzig die Sprache seines Exils bleibt ihm rudimentär erhalten ${ }^{5}$.

\footnotetext{
5 Freilich wird die Geschichte von Abel Nema in Alle Tage nicht so erzählt, wie es diese knappe Zusammenfassung der Romandiegese suggeriert, die Komposition und Ordnung der Erzählung ist um ein Vielfaches komplexer. Für eine ausführlichere Analyse vgl. KRAFT 2011: $183 \mathrm{ff}$.
} 


\title{
Vom jetzt und hier eines labyrinthischen Romans
}

Nennen wir die Zeit jetzt, nennen wir den Ort hier. (MORA 2004: 9)

Dieser erste und mittlerweile schon zu gewisser Berühmtheit ${ }^{6}$ gelangte Satz von Alle Tage erlaubt mindestens drei Lektüren. Zuvorderst verweist er auf eine (1) außerliterarische Konstellation. Das incipit erinnert daran, wie die Setzung eines diskursiven und deiktischen Startpunkts zugleich die Setzung einer Autorinstanz impliziert, die es ohne diesen Text nicht gäbe. Natürlich kann man sagen, dass die Autorin Terézia Mora durch den Erfolg ihres ersten Erzählbandes und die gewonnenen Preise in den Nuller Jahren bereits eine gewisse Bekanntheit erlangt hatte. Aber doch verweist dieser Romanauftakt auf eine psychologische und poetologische Konstellation, die dem frühen Schreiben, noch dazu dem „Willen zur Künstlerin“, der „Entstehung der Dichterin“ (MORA 2007: 73) vorgelagert ist. Eine Konstellation, die für die Autorin zu Beginn ihrer schriftstellerischen Karriere noch galt und an die in jenem ersten Satz gewissermaßen erinnert wird. In dem für ihre poetologischen Reflektionen wichtigen Text $»$ Die Dichterin in ihrer Zeit ${ }^{7}$ schreibt Terézia MoRA dazu:

\begin{abstract}
Mein erster Text [...] entstand 1997 - quasi aus dem Nichts heraus. Ein Nichts gibt es nicht? Jeder Text kommt von irgendwoher? Ja, aber. Wovon ich rede, ist jener vorbewusste Zustand, in dem zwar alles da ist, aber nichts einen Namen hat. [...] jener Zustand, in dem sich jemand vor dem Anfang, der das Wort ist, befindet. Vor ihrer Entstehung ist die Dichterin als Dichterin: nichts. Von diesem Nichts rede ich. Hier fangen wir an.

Bei mir dauerte diese Phase verhältnismässig lange. Ich war 26 Jahre alt, als ich meinen ersten Satz schrieb, und zwar nicht einfach so, weil ich gerade Bock darauf hatte, sondern weil ich ihn einfach nicht mehr nicht schreiben konnte. ,Literatur', schreibt Donald Barthelme, ,ist die Antwort auf einen Zwang und das Ergreifen einer Gelegenheit.' Als sich mir die Gelegenheit bot, ergab ich mich dem Zwang und sprach. (2007: 73)
\end{abstract}

Darüber hinaus darf dieser als »erstes Sprechen«(re-)inszenierte Anfangssatz als (2) kunstund literaturtheoretisch gut durchgewaschener Schöpfungsakt einer Erzählerinstanz gelesen werden, der ein Leitmotiv der Erzählung einführt. Denn als das Sprechen einer Stimme, die

\footnotetext{
${ }^{6}$ Eine vom 13. bis zum 15. März 2012 an der Universität Leipzig ausgerichtete Germanistentagung zu „Poetologien des deutschsprachigen Gegenwartsromans“ machte diesen Satz zu ihrem titelgebenden Motto (vgl. http://gegenwartspoetologien.wordpress.com/ (01/08/2012)).

${ }^{7}$ Unter diesem Titel sprach die Autorin 2006 im Rahmen der Tübinger Poetikdozentur sowie am 06. Juni 2007 zu Studierenden der Universität Duisburg-Essen (http://www.uni-due.de/de/presse/meldung.php?id=259 (01/08/2012)). Unter dem gleichen Titel veröffentlichte sie im März 2007 in der Neuen Zürcher Zeitung eine gekürzte Fassung der Tübinger Vorlesung, aus der hier zitiert wird.
}

Pandaemonium, São Paulo, v. 15, n. 20, Dez. /2012, p. $154-175$ - www.fflch.usp.br/dlm/alemao/pandaemoniumgermanicum 


\section{Kraft, T. - Poetik der Drastik}

aus dem Nichts ein deiktisches Etwas setzt ist jener Satz zugleich als Vorbote einer ganzen Kaskade christlicher Verweise im Verlauf des Romans zu verstehen, die nicht nur im Namen des Protagonisten, sondern auch in dessen gleich zu Beginn erzählten umgekehrten Kreuzigung an den Metallstangen eines Spielplatzes ihren (unheilvollen) Anfang findet; ein Anfang, der zugleich die größte proleptische Klammer der gesamten Erzählung darstellt, endet der Roman doch nur wenige Seiten (und Erzählsegmente) nach diesem, bereits zu Beginn erzählten Ereignis. Darüber hinaus suggeriert das unvermittelte hic et nunc ${ }^{8}$ (3) einen, durch die Überschrift „,0. JETZT Wochenende“ unterstrichenen, erzählerischen Nullpunkt, aus dem heraus sich eine zu erwartende stabile Deixis, also eine konkrete Bestimmtheit von Raum und Zeit ableiten ließe. Doch das jetzt und hier des Romanincipits ist keineswegs der temporale oder spatiale Startpunkt dieser Erzählung. Das gilt weder für seine diegetische Ordnung, noch für die semantische Entfaltung der Raum- und Zeitbezüge innerhalb der Geschichte des einsamen Abel Nema.

Diese Destabilisierung vermeintlich stabiler Setzungen gilt ebenso für die vom Text gesteuerte Lektüre, die auch aufgrund eines fehlenden Inhaltsverzeichnisses ${ }^{9}$ schon bald an Orientierung einbüßt. Erst mit fortschreitender Lektüre wird deutlich, dass der Roman einer hoch diffizilen Ordnung iterativer und ergänzender Anachronien folgt, deren Zusammenhang sich nur gelegentlich erschließt und für Momente zu einem kohärenten Verständnis der Handlungsabfolge verdichtet. So entsteht in immer neuen Volten ein Gefühl diegetischer Orientierungslosigkeit, Folge einer Überblendungstechnik, in der die Ort- und Zeitbezüge sowie das in Pro- und Analepsen geradezu ineinander erzählte Verhalten der Figuren die anfangs angekündigte Bestimmtheit der Null-Setzung gründlich widerlegen. Man blättert viel in Alle Tage, will man den Versuch unternehmen, die diskursive Chronologie mit der diegetischen abzugleichen. Kein Wunder, ziehen sich die ergänzenden Einschübe von wiederum in sich verschachtelten Rückblenden doch manchmal über mehr als hundert Seiten $^{10}$. Doch gerade dort, in der Verschränkung von Romangegenwart und Rückblende, gelingt es Mora, den Leser in kurze Phasen bekannter Lektüre zurückzuholen, welche dieser auf ähnliche Weise als vertraut zu erkennen glaubt, wie der Protagonist Abel einen Kirchturm,

\footnotetext{
${ }^{8}$ Diese Formulierung übernehme ich von Nathan Taylor, der auf der in Fn. 6 erwähnten Tagung einen Vortrag mit dem Titel „Realismus nach der (Post)Moderne? hic et nunc als ästhetisches Prinzip bei Terézia Mora“ hielt, von dem man sich einiges versprechen darf.

${ }^{9}$ Ein rekonstruiertes Inhaltsverzeichnis findet sich in KRAFT 2007: 111-112.

${ }^{10}$ Konstantins und Abels Wiedersehen in einem Park nach sieben Jahren, in denen sie sich nicht gesehen hatten, wird mit einem Satz auf S. 85 proleptisch anerzählt, um dann 248 Seiten später wieder aufgenommen und zu Ende erzählt zu werden.
} 


$$
\text { Kraft, T. - Poetik der Drastik }
$$

die Straßen zum Bahnhof oder einen Park (Mora 2004: 159), nur um von dort wieder abzutauchen in die multidirektionalen, ungewissen Seitenwege der Geschichte, so zuverlässig unzuverlässig geordnet wie die Großstadt, in der diese famos erzählte Geschichte spielt.

Das Buch legt mit versierter Eleganz Spuren aus, die so ziellos scheinen wie die Wege Abel Nemas durch die Stadt. Sie führen mal hierhin, mal dorthin. Moras Held ist mittendrin, aber nicht dabei. (JANDL 2004: 66)

Der metonymische Effekt einer erlebten Nähe zur verwirrenden Ort- und Orientierungslosigkeit des Protagonisten, der sich zwar in vollem Umfang zehn Sprachen, aber kaum den Verlauf zweier Straßenzüge merken kann, ist poetologisches Programm: „Wenn [...] Abel [...] anfängt umherzuirren, fängt auch der Satz an umherzuirren, man muss ihn nur lassen“ (MORA 2007: 73).

Dieses amnesische Erzählen ist der narratologisch leicht zu erfassende Vorbote einer Schicksalsgeschichte, in welcher die staatsbürgerliche Identität Abel Nemas, sein Herkunftsort, die Muttersprache, die Liebesfähigkeit, und schließlich auch der eigene Name verloren gehen. Dieses vielschichtige Verlusttrauma konkretisiert sich in der weitgehenden Abwesenheit territorial bestimmbarer Räume und gräbt sich analog $\mathrm{zu}$ Abels Orientierungslosigkeit tief in die Erzähl- und Figurenrede ein, wird doch beinahe jede toponymische Bestimmung im Roman unterdrückt, verschwiegen, verdrängt. Diese spezifische Form narrativer Amnesie artikuliert sich in Alle Tage in erster Linie im Modus einer lesergerichteten externen Fokalisierung, die schlichtweg das verschweigt, was gerade eigentlich gesagt wird; sie ist zugleich figurenpsychologisch deutbar, erzeugt sie doch einen durch die Lektüre erlebbar gemachten Wahrnehmungsfilter, welcher Abel Nema selbst zuzuschreiben ist.

Nach (wie vielen?) Jahren fragte Thanos seinen Stammgast: Wo kommst du her? Darauf antwortete er endlich was.

Verstehe, sagte Thanos. (MORA 2004: 258)

Abel war nur zufällig neben ihr stehen geblieben. Nur solange, bis er sich für eine Richtung entschieden hat: rechts oder links. Sie sprach die Muttersprache.

Wo seid ihr her? Fragte sie ihn nun direkt.

Erst sah es so aus, als würde er nicht antworten, dann antwortete er doch: Die anderen aus B., er selbst aus $\mathrm{S}$.

Nein! Aus S.? Wirklich? Sie schnappte nach Luft: Ich bin auch aus S.

Jetzt sah er sie an. (MORA 2004: 236) 


\title{
Kraft, T. - Poetik der Drastik
}

Die Unfähigkeit des Erzählers selbst, die initiale Setzung eines hier im jetzt der Erzählung zu konkretisieren, ist der narrative Abdruck eines Traumas, das in der erzwungenen Vertreibung aus einer verlorenen Heimat seine Wurzel hat. Was durch Flucht, Liebesentzug und Zusammenbruch der alten Ordnungen verloren gegangen ist, kann nicht kompensiert werden. So ist Abels Wurzellosigkeit keine Phase, sie ist ein Zustand. Als psychologische Disposition folgt ihm dieses Trauma auf jede seiner Stationen. Nie kommt er an einem eigenen Ort an, immer bleibt der Status seiner Existenz gebunden an eine von vorausgegangener Vertreibung geprägte Delokalisation, die selbst seinen sprachlichen Zugriff auf die Welt erfasst.

\section{Grenzen der Referentialität}

Deswegen ist alles, was er sagt, so, wie soll ich es sagen, ohne $O r t$, so klar, wie man es noch nie gehört hat, kein Akzent, kein Dialekt, nichts - er spricht wie einer, der nirgends herkommt. (MORA 2004: 13)

Abels einsilbiges Kommunikationsverhalten, diese Sprachlosigkeit des Sprachengenies, ist ein Leitmotiv des Romans. Die außergewöhnliche Fähigkeit zur Sprache verknüpft sich mit der Unfähigkeit zu sprechen. Schon Abels erster Wohngenosse Konstantin stellt diesen Zusammenhang etymologisch fest:

\begin{abstract}
Immer etwas etepetete, so ein Rührmichnichtan, aber du täuscht mich nicht, dein Name verrät dich: Nema, der Stumme, verwandt mit dem slawischen Nemec, heute für: der Deutsche, früher für jeden nichtslawischer Zunge, für den Stummen also, oder anders ausgedrückt: den Barbaren. Abel, der Barbar, sagte eine Frau namens Kinga und lachte. Das bist du. (MORA 2004: 14)
\end{abstract}

Die mehrdeutige Etymologie, die sich in noch viel komplexerer Form für seinen Vornamen auffächern lässt ${ }^{11}$, verweist zugleich auf das Ursprungsproblem dieser Figur, auf die Leere, die sich auf dem Weg zu den Wurzeln auftut. Diese Wurzellosigkeit entfaltet eine komplexe Poetik der Ortlosigkeit, die Alle Tage nicht nur in den Deiktika, Anachronien und Ellipsen des narrativen Diskurses entwickelt (s.o.). Darüber hinaus modelliert der Roman einen Typus des unzuverlässigen Erzählens, der nicht auf eine Erzählerinstanz selbst zurückgeführt werden kann und der das innerliterarische Referenz-System aus den Angeln hebt. Es ist Abels

\footnotetext{
${ }^{11}$ Vgl. zur Alias-Situation Abel Nemas JANDL 2004: 66, KRAFT 2007: 47ff., sowie KRAFT 2011: 159ff..
} 


$$
\text { Kraft, T. - Poetik der Drastik }
$$

Präsenz im Text, seine amnesische Wahrnehmung der Welt, die ein unzuverlässiges Erzählen provoziert.

\begin{abstract}
Manchmal ging er vor dem Labor in die Originalfassungen verschiedenster Filme, Übungszwecke, manchmal kaufte er sich an einer eventuell vorhandenen Theke etwas zu essen oder zu trinken: indem er darauf zeigte. (MORA 2004: 105)
\end{abstract}

Man kann es leicht überlesen, aber die eventuell vorhandene Theke enthält eine ganze Kunstreflexion, lässt sich diese Passage doch als metaleptischer Ein-Wort-Kommentar zum Repräsentationsdilemma der Künste lesen. Die deiktische Destabilisierung aller Raumbezüge vermischt sich unauflöslich mit der poetologischen Reflexion zum unmöglichen Unterfangen referentieller Verbindlichkeit.

Wenn es stimmt, dass Literatur als weiterhin gültiges Leitmedium einer ästhetischen Arbeit an und mit der Sprache darin ,,spezialisiert ist, weder diskursiv noch disziplinär noch als Dispositiv kulturellen Wissens spezialisiert zu sein“ (ETTE 2012: 4), folglich Phänomene, Fragen und Probleme unserer Lebenswelt in ein spezifisches, literarisches Wissen transformieren kann, dann müsste über dem Roman Alle Tage die Frage stehen: Wie können wir Fremdheit literarisch denken? Terézia Moras Antwort ist die ebenso literarische wie metaliterarische Figur Abel Nema. Er ist der Andere, jener Unvereinbare, der sich auch dadurch einer sprachlichen Verortung in der Diegese des Romans verweigert, weil er dessen Realitätsannahmen überschreitet.

\begin{abstract}
Was in diesen drei Tagen in Abel Nemas Gehirn vor sich ging, lässt sich nicht genau erfassen. Er selbst hat keine Erinnerung daran, lediglich eine Vorstellung davon. Etwas in der Art, als hätte ein Jemand die einzelnen Teile eines Schiebespiels so lange hin und her geschoben, bis sich ein völlig neues Bild ergab. So organisierte etwas, so organisierte sich das Labyrinth in Abel Nemas bis dahin in allen Schulfächern gleichermaßen begabten und desinteressierten Verstand so lange um, bis alles, was bis dahin eine Rolle gespielt hatte, das Gewusel von Erinnerung und Projektion, Vergangenheit und Zukunft, das die Gänge verstopfte und in den Zimmern lärmte, irgendwo verstaut war, in geheimen Wandschränken, und er, nun leer, bereit zur Aufnahme einer einzigen Art von Wissen: von Sprache. Dies ist das Wunder, das Abel Nema widerfahren ist. (MORA 2004: 74-75)
\end{abstract}

Das ihm zugekommene Wunder, die Unerklärbarkeit seiner kognitiven wie performativen, linguistischen Fähigkeiten ist der dafür offensichtlichste Beleg. Als ästhetischer Bruch mit den Realitätskonventionen der Diegese ist das Wunder Ausdruck einer Inkompatibilität zwischen Individuum (Abel) und Welt (Romandiegese), die sich konkretisiert in Abels 


$$
\text { Kraft, T. - Poetik der Drastik }
$$

Sprachlosigkeit. Er ist, „nun leer“, weniger eine tatsächliche Figur des Romans, vielmehr eine dem Roman entgleitende Denkfigur. Seine Existenz ist in dieser eventuell vorhandenen Welt nicht gesichert.

\section{Orientierungslose Glottophagie}

Abels totale Konzentration auf das möglichst perfekte Erlernen möglichst vieler Sprachen scheint für den Ort- und Heimatlosen das einzige, klare Bezugssystem zu sein: die subjektlose, reine Sprache wird erlernt, weil dem Sprechen selbst keine Bedeutung mehr zukommt. Indem „das Wunder“ Abels Sprachen entpersonalisiert, sie aller Individualität entkernt, eingeschlossen der eigenen, idiomatischen Herkunft, wird zugleich die sprachlich gespeicherte eigene Vergangenheit, das Erinnern in den Idiosynkrasien des eigenen Lebens ausgeblendet. Jenseits der mnemotechnischen Selbstvergewisserungen des eigenen Ideolekts einer Herkunftsprache steht die erinnerungslose Mathematik des Sprachsystems. Die Sehnsucht eines stetig Fremden, in nichts mehr fremd und damit auch in nichts mehr eigen zu sein, führt zu einem wundersamen Vielsprachenkenner, der nirgends zuhause ist. Von hier zum nächsten Schritt, nämlich der kategorialen Infragestellung jeglicher Referentialität von Sprache außerhalb ihrer selbst, ist es nicht weit.

Früher wollte Abel, oder wer weiß, er schickte sich an, Geographielehrer zu werden, jetzt war das Innere seines Mundes das einzige Land, dessen Landschaften er bis ins Letzte kannte. [...] Sage und schreibe vier Jahre lang, nach Männerwohnheim, Linoleum, Neonlichtern riechende Zeit, bewegte er sich so gut wie ausschließlich entlang einer einzigen Strecke: vom Wohnheim zum Sprachlabor und zurück. [...] Er ging nirgends hin, wo er nicht unbedingt hin musste, auch ins Labor ging er meist nachts, wenn er allein sein konnte. [...] Das macht die ganze Sache noch ein Stück unglaublicher, um nicht zu sagen unheimlich, sagte man in den Fremdsprachigen Philologien. Er lernt Ton um Ton, analysiert Frequenzzeichnungen, wühlt sich durch die Codes der Lautschrift und färbt sich die Zunge schwarz, um die Abdrücke zu vergleichen. Auf die Dauer schmeckt das wie Strafe. Als hätte man Tinte oder Waschpulver gegessen. Da wird einem die Bezeichnung Labor erst so richtig vor Augen geführt, Technik ist primär, Mensch ist sekundär. Als züchtete er des Nachts dort seinen Homunculus, nur dass dieser hier ganz aus Sprache besteht, der perfekte Klon einer Sprache zwischen Glottis und Labia. (MORA 2004: 100-101)

Abel Nemas ganz private Glottophagie ist direkt geknüpft an die Auslöschung von Schrift als Träger von Bedeutung. Schrift ist lediglich der Code eines Lautes und als solcher Träger rein akustischer, nicht semantischer Information. Die subtile und weiblich fokalisierte Erotik, die 


$$
\text { Kraft, T. - Poetik der Drastik }
$$

sich in dieser Passage zu Abels technizistischem Spracherwerb in dem Verlangen äußert, sich Sprache als Schrift mit der Zunge einzuverleiben und diese „zwischen Glottis und Labia“ zu erzeugen, bleibt letzlich Projektion. Abel mag diese Vorstellungen in seiner weiblichen Umwelt provozieren, doch sie erfüllen kann er nicht. Denn was er mit Sprache, Zunge und Mund tut, erfüllt keinen über den Erwerb hinausgehenden Zweck. Als ihn Kinga in der Sylvesternacht auf seine schwarzen Zähne anspricht, sagt Abel lediglich: „das ist ein Verfahren der Phonologie“ (MORA 2004: 138). Die Antwort verweist erneut auf die kognitive Trennung zwischen Sprachkompetenz und Lauterwerb, die für Abels wundersames Sprachtalent so bezeichnend ist. Seine perfekt geklonten Sprachen sind als soziale Kommunikationsinstrumente eben nicht mehr lebensfähig.

\section{Gattungstraditionen auf Entzug}

Gattungstypologisch lässt sich Alle Tage in der Tradition des Entwicklungsromans lesen und steht auch damit in einer an die Gattungsnormen des bürgerlichen Realismus anknüpfenden Linie. Doch natürlich gibt es einen entscheidenden Unterschied, erschließt sich doch die Entwicklung der Hauptfigur nicht als Chronologie von Ereignissen und Entscheidungen, sondern wird in einem einzigen, formal wie narrativ vom Rest des Buches abgegrenzten Kapitel „Zentrum: Delirium“ als monologischer Bewusstseinsstrom komprimiert. Das „Zentrum“-Kapitel ist in jeder Hinsicht erzählerischer Zenit, Sonderfall und Kontrapunkt zum Rest des Romans. Diegetisch schließt es die Lücke zwischen den zwei Präsenszeiten der Rahmenerzählung, zugleich bricht es als Erzählung mit allen Konventionen der bisherigen Narration. Es erzählt, in über fünfzig, durch keinerlei Unterkapitel gegliederten Seiten Abels viertägigen Drogenrausch, ausgelöst durch eine Überdosis Fliegenpilze, als eine Mischung aus halluzinogenen Alb- und Erlösungsträumen und weiss sich damit in einer Tradition der Moderne, die nach kanonischem Verständnis bei Joyce begann. Ihm huldigt Mora bereits im Prolog zu Alle Tage mit einem (allerdings nicht ausgewiesenen) Zitat (MORA in KRAFT 2007: 107).

Eine Auflösung von Abels Lebensproblematik kann nur auf der einzig konsequent und bruchlos durcherzählten Motivfolie des gesamten Textes verhandelt werden: des Traumas durch Krieg, Vertreibung und Gewalt. Somit ist die Gattung hier - wie kann es anders sein mehr historisches Zitat als Erfüllung einer gattungsspezifischen Form. Das Telos der 


$$
\text { Kraft, T. - Poetik der Drastik }
$$

Erzählung ist die Ankunft in einer neuen sozialen Ordnung, der der Kleinfamilie, unter Preisgabe seiner Sprache sowie aller seiner besonderen Fähigkeiten. Auf dieses Ziel strömt die Handlung des Romans allerdings ohne jegliche Handlungsmotivation ihres Protagonisten hin. Die ,Bildung’ Abel Nemas zu einem gesellschaftsfähigen Menschen findet nicht statt. So sehr wie sich Abel Nema einem Begriff entzieht, tut dies der Roman in einem gattungstheoretischen Sinne. Zwar behauptet Alle Tage zunächst die Form des Bildungs- oder Entwicklungsromans, widerstrebt dessen teleologischer Ausrichtung aber, auch wenn der Roman dessen Absichten oberflächlich auf den letzten Seiten einzulösen und das gattungshistorische Postulat, „dass Ziel der Entwicklung des Individuums die Selbstfindung in einer Versöhnung mit der Welt sein sollte“ (JACOBS 2009: 60), auszufüllen scheint.

Damit ist der Roman die Geschichte einer fundamentalen Desillusionierung, womit er sich erneut in die Tradition des französischen Realismus einschreibt (Stendhals Le Rouge et le Noir, Balzacs Illusions perdues, Flauberts Éducation sentimentale, vgl. u.a. KÖHLER 2006). Abel Nema scheitert als „Fremder an sich“ (MORA in AYATA u. a. 2005: 30) an der Gesellschaft, in die er aufgrund äußerer Schicksale hineingeworfen wird und von der er eigentlich nie etwas Spezifisches wollte. Die Desillusion speist sich nicht aus einem gescheiterten Aufstieg à la Julien Sorel, sondern aus der Unauflöslichkeit von Fremdheit. Moras Erzähltechnik bedient sich dabei dem seit Flaubert zum narrativen Sortiment der Weltliteratur gehörenden Modus der erlebten Rede, verknüpft diese aber zugleich mit einer multifokalen Steuerung von Information und Perspektive, welcher schließlich die Verlässlichkeit des Erzählens selbst abhanden kommt. Der Flux der zuweilen intersyntaktischen Fokalisierungswechsel produziert das ästhetische Erlebnis disruptiver Einbrüche von Fremdwahrnehmung in den kohärenzstiftenden Fortlauf der Eigenwahrnehmung. Die umfassende Orientierungslosigkeit der Figuren, vor allem Abel Nemas selbst, auf der Ebene konkreter geographischer wie diegetischer Ordnungsmuster entspricht auf der Ebene kategorialer Bestimmungen die Figur des Entzugs, die im Falle Abel Nemas sogar die Grenzen der Romandiegese in Metalepsen, die manchmal nur aus einem Wort bestehen, überschreitet.

Diese Pendelfigur zwischen Behauptung und Wiederlegung kategorialer Eindeutigkeit lässt sich auch gendertheoretisch begründen. Strebt die Entwicklung im traditionellen Bildungsroman darauf hin, das „engendering of the male Subject“ im Kontext patriarchalischer symbolischer Ordnungen zu erzählen (John H. SMITH in JACOBS 2009: 61), ist die sexuelle Orientierung Abel Nemas ebenfalls mehr Orientierungslosigkeit als klärende 


$$
\text { Kraft, T. - Poetik der Drastik }
$$

Entwicklung. Zwischen asexuellem Desinteresse an seiner weiblichen wie männlichen Umgebung und homosexuellen Nachtclubeskapaden mit latent pädophiler Ausrichtung beweist er sich zugleich als emphatische Vaterfigur im Umgang mit Omar, Mercedes' hochintelligentem Sohn, mit dessen Mutter er schließlich zum Ende des Romans doch noch und offenbar aus freien Stücken - ein Kind zeugt. Und dennoch liegt gerade in dieser Ununterscheidbarkeit vormals als stabil behaupteter Kategorien genau die künstlerischsprachliche wie epistemische Relevanz dieses Romans, der den alten und von den Figuren des Romans immer wieder geäußerten Wunsch nach Fixierbarkeit ad absurdum führt und sprachlich wie formell in zahlreichen Metonymien durchspielt. Im Sinne eines „engendering“ geht dieser Werdegang nicht auf, Abels Verhalten entzieht sich den gängigen Kategorien von Geschlechtsidentität und stellt sie queer.

\section{Zusammenfassung und Ausblick}

Die Antwort auf die Frage, wie Fremdheit in Zeiten fortgeschrittener und fortschreitender Globalisierung literarisch gedacht und verstanden werden kann, ergibt sich mit Blick auf Terézia Moras Roman Alle Tage nicht nur aus den Innenperspektiven oder Handlungsmotivationen der Hauptfigur. Sie ergibt sich ebenso aus den metaliterarischen Verfahren des Romans. Indem der Roman ein Bedürfnis nach deiktisch eindeutiger Referenzialität auf narrativer wie diskursiver Ebene sowohl provoziert als auch verweigert, verweist er nicht nur auf die eigene Literarizität im Sinne eines fiktionalen und entpragmatisierten Diskurses, sondern erzählt sich selbst als Modell, nicht als Abbild. Alle Tage ist eben auch Alle Orte und, wie die Autorin selber sagt: „das Leben“ (MEYER-GosAU 2004). Der produktionsästhetische Ausgangspunkt dieser Arbeit am Modell des Lebens unter den Vorzeichen von Fremdheit und Traumatisierung ist bei Terézia Mora eine an die Traditionen realistischen Schreibens anknüpfende Poetik, die das Abbildungsprimat des 19. Jahrhunderts auf Entzug stellt und sich zugleich einer intrinsischen Motivation verpflichtet sieht, die bestrebt ist, mit den Mitteln der Literatur unser Nachdenken über Prozesse der Globalisierung kritisch zu reflektieren.

Im Sinne einer Poetik der Drastik antwortet Mora auf dieses theoretisch, semantisch und ideologisch aufgeladene Feld mit einer Leerstelle. In Alle Tage ist diese Leerstelle Abel Nema. Er ist zugleich Protagonist wie (Anti-)Held der Globalisierung, Personifikation des 


$$
\text { Kraft, T. - Poetik der Drastik }
$$

Traumas von Migration und Krieg. Im Unterschied zu Darius Kopp, dem Protagonisten aus Moras zweitem Roman Der einzige Mann auf dem Kontinent, reflektiert Abel durchaus seinen Zustand, dieses »etwas-nicht-Sein« im Angesicht eines ebenso multiplen wie irreversiblen Verlustes. Aber weder erlaubt ihm diese Reflektion einen Ausweg aus seinen Verhältnissen, noch legt dieser Proto-Vertriebene zu irgendeinem Zeitpunkt jene Fremdheit ab, die ihn für seine Umwelt zu einer so anziehenden wie irritierenden Person macht.

Die leeren oder nur angedeuteten Signifikanten der Romantopographie sowie des historischen Kontextes, in dem sich die Diegese von Alle Tage entfaltet, sind Ausdruck eines amnesischen Erzählens, das seine Realistik im Sinne einer plausiblen Darstellungsweise in der durch Abel modulierten Fokalisierung finden. Als „leeres Zentrum“ (MoRA 2004: 107) provoziert seine Präsenz im Text die Lücken im narrativen Diskurs. Auch er ist als Figur der für die Tradition des realistischen Romans typische Fall eines modèle réduit, zu dessen Funktion Peter Brooks schreibt:

Emma Bovary and Dorothea Brooke, Old Goriot and Nana - such characters have taken on an imaginative reality in their cultures, they are referred to as if they were real, or rather, more significant than the merely real, since they sum up and represent more fully certain choices of ways of being. They offer, in the best possible sense, criticisms of life: instances that lend themselves to discussion and debate, that pose important questions about our being in the world. (BROOKS 2005: 5)

Natürlich haben wir es bei Terézia Mora nicht mit einem Wiederaufguss einer Poetik des 19. Jahrhunderts zu tun. Doch repräsentiert Moras Schreiben und die in ihren zahlreichen Epitexten artikulierte Poetik ein kritisches Bewusstein für eine Welt, die sich in ähnlich grundlegender Weise wie jene des 19. Jahrhunderts (vgl. OsteRHAMMEL 2009) zu verwandeln scheint. Welche Lebensentwürfe erlaubt, besser: provoziert unsere Zeit? Welches Leben ist möglich in einer Zeit, deren neue Unübersichtlichtkeit nach Postmoderne und im Sog fortgesetzter Globalisierungsschübe einfache teleologische Auflösung unterbindet? Diesen Fragen geht Terézia Mora in ihren bisher zwei Romanen, von denen in dieser Untersuchung nur der erste behandelt werden konnte, poetisch und poetologisch anspruchsvoll nach. Und wenn Hans Vilmar Geppert, wie Moritz Baßler schreibt,

damit recht hat, die Suche des ,realistischen Wegs' gegen die systemisch angelegten Experimente und schließlich der Moderne zu stellen, dann läge das Aufregende dieses Realismus in Lösungsversuchen innerhalb einer niemals vollständig verstandenen Welt, innerhalb einer konstitutiven Unübersichtlichkeit. Gegen das Von-Grund-aufNeu-und-Besser-Machen der Moderne als ihre Hybris und ihr totalitärer Zug erscheint 


$$
\text { Kraft, T. - Poetik der Drastik }
$$

das Durchwurschteln der realistischen Helden als Operieren im immer schon Vorgegebenen, nie grundsätzlich Infragezustellenden, als Alternative, die womöglich im Realismus der Postmoderne wieder aktualisiert wird. (BAßLER 2007: 449)

\section{Literaturverzeichnis}

AUST, Hugo. Literatur des Realismus. 3., überarbeitete und aktualisierte Auflage. Stuttgart, Weimar, J. B. Metzler, 2000.

AyATA, Imran/KAMINER, Wladimir/KeRMANI, Navid/MoRA, Terézia. „Ich bin ein Teil der deutschen Literatur, so deutsch wie Kafka“. Interview mit Terézia Mora, Imran Ayata, Wladimir Kaminer und Navid Kermani. In: Literaturen (4), 2005, 26-31.

BaCHMANN, Ingeborg. Die gestundete Zeit. Gedichte [1957]. München, Zürich, Pieper, ${ }^{4} 1985$.

BAßLER, Moritz. Moderne und Postmoderne. Über die Verdrängung der Kulturindustrie und die Rückkehr des Realismus als Phantastik. In: BECKER, Sabine/KIESEL, Helmuth (ed.): Literarische Moderne: Begriff und Phänomen. Berlin, New York, de Gruyter, 2007, 435-450.

BrooKs, Peter. Realist Vision. New Haven, London, Yale University Press, 2005.

DATH, Dietmar. Die salzweißen Augen. Vierzehn Briefe über Drastik und Deutlichkeit. Frankfurt am Main, Suhrkamp, 2005.

Dethloff, Uwe. Französischer Realismus. Stuttgart, Weimar, J. B. Metzler, 1997.

ETTE, Ottmar. TransArea. Eine literarische Globalisierungsgeschichte. Berlin, Boston, de Gruyter, 2012.

GuPTA, Suman. Globalization and literature. Cambridge, Polity, 2009.

HARDER, Matthias (ed.). bestandsaufnahme. Deutschsprachige Literatur der neunziger Jahre aus interkultureller Sicht. Würzburg, Königshausen \& Neumann, 2001.

HÜBNER, Klaus. Chamisso - wohin? Ein Rückblick auf ein Symposium in Marbach. In: chamisso. Viele Kulturen - eine Sprache (März), 2010, 18-20.

JACOBS, Jürgen C. Bildungsroman. In: LAMPING, Dieter (ed.). Handbuch der literarischen Gattungen. Stuttgart, Alfred Kröner Verlag, 2009, 56-64.

JANDL, Paul. Wunder zwischen Abel und Babel. „Alle Tage” - Terézia Moras unheiliger Roman über das Fremde. In: Neue Zürcher Zeitung. Zürich, 05/10/2004, 66.

KAROw, Matthias/WinkLer, Bastian. Phon: Dietmar Dath. Eine praktische Frage. In: bella triste. Zeitschrift für junge Literatur (14), 2006, 62-66.

KLEIN, Wolfgang. Realismus / realistisch. In: BARCK, Karlheinz/FONTIUS, Martin/SCHLENSTEDT, Dieter/STEINWACHS, Burkhart/WOLFZETTEL, Friedrich (ed.): Ästhetische Grundbegriffe $(\ddot{A G B})$. Band 5: Postmoderne - Synästhesie. Studienausgabe. Stuttgart, Weimar, J. B. Metzler, 2010, 149-197.

KÖHLER, Erich. Vorlesungen zur Geschichte der Französischen Literatur. Das 19. Jahrhundert II [1987]. Digitale Bearbeitung. Herausgegeben von Dietmar Rieger. Freiburg im Breisgau, Universitätsbibliothek $\quad$ Freiburg, $2006 . \quad$ http://www.freidok.unifreiburg.de/volltexte/2652/pdf/19 Jahrhundert 2.pdf (01/08/2012).

KRAFT, Tobias. Literatur in Zeiten transnationaler Lebensläufe: Identitätsentwürfe und Großstadtbewegungen bei Terézia Mora und Fabio Morábito. Magisterarbeit. Universität Potsdam, Institut für Germanistik, Institut für Romanistik, Potsdam, 2007. http://opus.kobv.de/ubp/volltexte/2007/1295/ (01/08/2012). 


$$
\text { Kraft, T. - Poetik der Drastik }
$$

KRAFT, Tobias. Verortungsbedarf und Ortlosigkeit als Dauerzustand innerer und äußerer Migrationen. Bewegungsanalytische Überlegungen und narratologische Exkursionen am Beispiel von Terézia Moras Transitroman „Alle Tage“ (2004). In: FRANZ, Norbert/KunOw, Rüdiger (ed.). Kulturelle Mobilitätsforschung: Themen - Theorien - Tendenzen. Potsdam, Universitätsverlag Potsdam, 2011, 169-209.

MAgENAU, Jörg. Mensch ohne Menschheit. In: taz. die tageszeitung, literataz. Berlin, 06/10/2004, 9.

MEYeR-GosAU, Frauke. Böse Erlösung. Ein Nachmittag und Abend mit Terézia Mora in Barcelona anlässlich des Erscheinens ihres ersten Romans „Alle Tage“. In: Literaturen (9), 2004, 44-48.

MORA, Terézia. Alle Tage. Roman. München, Luchterhand Literaturverlag, ${ }^{5} 2004$.

MORA, Terézia. Seltsame Materie. Erzählungen [1999]. Reinbek bei Hamburg, Rowohlt Taschenbuch, ${ }^{2} 2005$.

MORA, Terézia. Über die Drastik. In: bella triste. Zeitschrift für junge Literatur (16), 2006, 68-74.

MorA, Terézia. Die Dichterin in ihrer Zeit. In: Neue Zürcher Zeitung. Zürich, 10-11/03/2007, 73.

OSterhammel, Jürgen. Die Verwandlung der Welt. Eine Geschichte des 19. Jahrhunderts. München, C.H. Beck, ${ }^{2} 2009$.

RECKWITZ, Erhard. Realismus-Effekt. In: NÜNNING, Ansgar (ed.): Metzler-Lexikon Literatur- und Kulturtheorie. Ansätze - Personen - Grundbegriffe. 4., aktualisierte und erw. Aufl. Stuttgart, Metzler, 2008, 609-610.

REICHARDT, Ulfried. Globalisierung. Literaturen und Kulturen des Globalen. Berlin, Akademie Verlag, 2010.

STOCKHAMMER, Robert. DFG-Graduiertenkolleg "Funtionen des Literarischen in Prozessen der Globalisierung. Darstellung des Forschungs- und Studienprogramms. München, 2011. http://www.graduatecenter-lmu.de/global/images/stories/pdf/grako.pdf (01/08/2012)

STOPKA, Katja. Aus nächster Nähe so fern. Zu den Erzählungen von Terézia Mora und Judith Hermann. In: HARDER, Matthias (ed.): bestandsaufnahme. Deutschsprachige Literatur der neunziger Jahre aus interkultureller Sicht. Würzburg, Königshausen \& Neumann, 2001, 147166.

WeIDERMAnN, Volker. Aus einer anderen Welt. Im Vergleich zu ihr sind alle anderen gleich: Die ungarisch-deutsche Schriftstellerin Terézia Mora hat ihren ersten Roman geschrieben. In: Frankfurter Allgemeine Sonntagszeitung, Frankfurt am Main, 08/08/2004, 27. 